Т. П. Водопьянова, В. С. Жуковень

Белорусский государственный технологический университет

\title{
МЕТОД АНАЛИЗА ИЕРАРХИЙ КАК ИНСТРУМЕНТ УПРАВЛЕНИЯ РИСКАМИ ИННОВАЦИОННОЙ ДЕЯТЕЛЬНОСТИ ПРЕДПРИЯТИЙ
}

В статье рассматривается применение метода анализа иерархий с целью управления рисками инновационной деятельности предприятия по производству мебели. Если перед предприятием стоит задача выбора оборудования для внедрения нового вида продукции и при этом необходимо минимизировать риски и не допустить снижения спроса на продукцию, метод позволяет оценить оборудование по наиболее важным критериям.

Процедуры расчетов метода анализа иерархий достаточно просты, а сам метод является универсальным, поскольку схема применения не зависит от сферы деятельности, в которой принимается решение. При этом могут использоваться абсолютно разные критерии с различным уровнем их значимости, что позволяет оценивать любой вид риска.

Для принятия решения по выбору оборудования рассмотрены следующие критерии: объем загрузки пиломатериалов, мощность электродвигателя, продолжительность сушки.

Ключевые слова: риск, инновационная деятельность, метод анализа иерархий.

Для цитирования: Водопьянова Т. П., Жуковень В. С. Метод анализа иерархий как инструмент управления рисками инновационной деятельности предприятия // Труды БГТУ. Сер. 6, История, философия. 2021. № 1 (245). С. 181-185.

\section{T. P. Vodop'yanova, V. S. Zhukoven'}

Belarusian State Technological University

\section{HIERARCHY ANALYSIS METHOD AS A RISK MANAGEMENT FOREIGN ECONOMIC ACTIVITY}

The article discusses the application of the method of analysis of hierarchies in order to manage the risks of innovative activity of a furniture manufacturing enterprise. If the company is faced with the task of selecting equipment for the introduction of a new type of product and at the same time it is necessary to minimize risks and prevent a decrease in demand for products, the method allows you to evaluate the equipment according to the most important criteria.

The calculation procedures of the hierarchy analysis method are quite simple, and the method itself is universal, since the application scheme does not depend on the field of activity in which the solution is applied. In this case, completely different criteria can be used with different levels of their significance, which allows you to assess any type of risk.

To make a decision on the choice of equipment, we considered the following criteria: the volume of loading of lumber, the power of the electric motor, the duration of drying.

Key words: risk, innovative activity, hierarchy analysis method.

For citation: Vodop'yanova T. P., Zhukoven' V. S. Hierarchy analysis method as a risk management foreign economic activity. Proceedings of BSTU, issue 6, History, Philosophy, 2021, no. 1 (245), pp. 181-185 (In Russian).

Введение. Инновационный процесс можно определить как рискованный процесс последовательного превращения идеи в товар, проходящий стадии фундаментальных и прикладных исследований, конструкторских разработок, маркетинга, производства, сбыта.

Возможность потерь и неудач в инновационной сфере значительно выше, чем во всех остальных. Инновационный риск - это вероятность убытков, имеющих место при вложении средств в производство новых товаров и услуг, которые, возможно, не найдут ожидаемого спроса на рынке. Инновационный риск возникает в следующих ситуациях:
- при внедрении более дешевого метода производства товара или услуги по сравнению с уже использующимися;

- при создании нового товара или услуги в связи с использованием физически и/или морально устаревшего оборудования;

- при производстве нового товара или услуги с помощью новой техники и технологии, при этом существует угроза не найти потребителя.

Реагирование на риск связано с анализом минимизации потерь и их возмещением.

Основная часть. Выбор оборудования следует осуществлять по различным критериям и 
для этого может применяться метод анализа иерархий - МАИ, разработанный математиком Т. Саати [1].

Суть метода анализа иерархий состоит в декомпозиции проблемы выбора на простые составляющие части и обработке суждений лица, принимающего решение. В результате определяется значимость исследуемых альтернатив для всех критериев, находящихся в иерархии. Относительная значимость выражается численно в виде векторов приоритетов. Полученные значения векторов приоритетов являются оценками в шкале отношений, позволяющими сделать количественно обоснованный выбор, принять решение [1].

Цель метода анализа иерархий - обоснование выбора наилучшей из предлагаемых альтернатив, характеристики которых являются векторами с разнородными, в том числе и с нечетко определенными, отдельными компонентами [2].

Этапы применения МАИ: 1) представление проблемы в виде иерархии (цели, ранжирование критериев и перечень альтернатив); 2) попарное сравнение критериев по важности по девятибалльной шкале с составлением соответствующей матрицы (таблицы) размера $(n \times n) ; 3)$ определение множества векторов приоритетов для элементов каждого уровня; 4) подсчет количественного индикатора каждой альтернативы, оценка эффективности, принятие управленческого решения [1].

Система парных сведений приводит к результату, который может быть представлен в виде обратно симметричной матрицы. Элементом матрицы $a(i, j)$ является интенсивность проявления элемента иерархии $i$ относительно элемента иерархии $j$, оцениваемая по шкале интенсивности от 1 до 9, где оценки имеют следующий смысл: равная важность - 1; умеренное превосходство - 3; значительное превосходство - 5; сильное превосходство - 7; очень сильное превосходство - 9; в промежуточных случаях ставятся четные оценки: $2,4,6,8$ (например, 4 - между умеренным и значительным превосходством).

Собственный вектор элементов каждого уровня матрицы $(V)$ вычисляется как геометрическая средняя: извлекается корень $n$-й степени ( $n$ - размерность матрицы сравнений) из произведений элементов каждой строки. Например, формула для расчета собственного вектора элемента $k_{n}$ матрицы сравнений для критериев имеет вид [3]:

$$
V_{n}=\left(\left(k_{n} / k_{1}\right) \cdot\left(k_{n} / k_{2}\right) \cdot \ldots \cdot\left(k_{n} / k_{n-1}\right) \cdot 1\right)^{1 / n} .
$$

Нормированный вектор приоритетов $(W)$ определяется как доля (вес) собственного вектора элементов каждого уровня матрицы $(V)$ в общей совокупности собственных векторов матрицы. Например, формула для расчета нормированного вектора приоритетов собственного вектора элемента $W_{n}$ матрицы сравнений для критериев имеет вид

$$
W_{n}=\frac{V_{n}}{\sum_{i=1}^{n} V_{i}} .
$$

Синтез полученных коэффициентов важности осуществляется по формуле

$$
S_{j}=\sum W_{i} \cdot V_{j i},
$$

где $S_{j}$ - показатель качества $j$-той альтернативы; $W_{i}-$ вес $i$-того критерия; $V_{j i}-$ важность $j$-той альтернативы по $i$-тому критерию.

Оценка альтернатив осуществляется по максимальному значению $S_{j}$, принимается наиболее предпочтительное, эффективное управленческое решение.

ОАО «Стройдетали» специализируется на производстве межкомнатных деревянных дверей не только из цельного массива хвойных пород и ольхи, но и облицованных натуральным шпоном, шпоном файн-лайн. При непосредственном участии высококвалифицированных специалистов акционерное общество динамично развивается в перспективных инновационных направлениях (выпуск топливных брикетов, садовых и дачных домиков и гаражей из профилированного бруса хвойных пород), осваивая при этом новое оборудование, новые материалы, новые технологии.

Важным направлением инновационного менеджмента ОАО «Стройдетали» является модернизация сушильной камеры. При ее выборе следует учесть технологические риски: из-за неравномерности просыхания пиломатериалов в штабеле, потери древесиной своего естественного цвета, большой продолжительности процесса сушки, высокого энергопотребления ухудшается качество пиломатериалов, появляются дефекты и соответственно это приводит к рискам в сфере конкурентоспособности продукции.

Критериями, которые помогут в выборе наилучшего варианта сушильной камеры, являются объем загрузки пиломатериала, м $^{3}$ мощность электродвигателя внутрикамерного вентилятора, кВт/ч; продолжительность сушки пиломатериала, сут.

Данные критерии были выбраны не случайно, так как от объема загрузки и продолжительности сушки будет зависеть продолжительность процесса изготовления готовой продукции. Когда на предприятие поступит крупный заказ, сушильные камеры могут не справиться с тем объемом пиломатериалов, который нужно 
будет высушить. А от мощности электродвигателя внутрикамерного вентилятора зависит потребление электроэнергии, чем ниже потребление, тем ниже затраты на сушку пиломатериалов.

На основании вышеперечисленных данных составим иерархию для нашей проблемы на рис. 1. Наибольший вес имеет продолжительность производственного процесса, поэтому наиболее важным критерием будет продолжительность сушки $k_{3}$ (табл. 1). Чем меньше его значение, тем быстрее пиломатериалы будут отправлены на следующий производственный процесс.

Вторым критерием по важности будет выступать объем загрузки пиломатериалов $k_{1}$, так как при быстром цикле сушки можно чаще загружать меньшие объемы пиломатериалов.
И последним по важности критерием является мощность электродвигателя $k_{2}$.

Построим матрицу парных сравнений критериев (табл. 2). Отношение согласованности составляет 2,6\%. Для использования в дальнейших подсчетах, значение отношения согласованности должно находиться в пределах 10\%.

Составим табл. 3 числовых оценок матрицы парных сравнений альтернатив по влиянию критерия $k_{1}$. Как видно из табл. 3, индекс согласованности равен 0,01 , отношение согласованности составляет $1,7 \%$.

В табл. 4 числовых оценок матрицы парных сравнений альтернатив по влиянию критерия $k_{2}$ (мощности электродвигателя) отношение согласованности составляет $1,7 \%$.

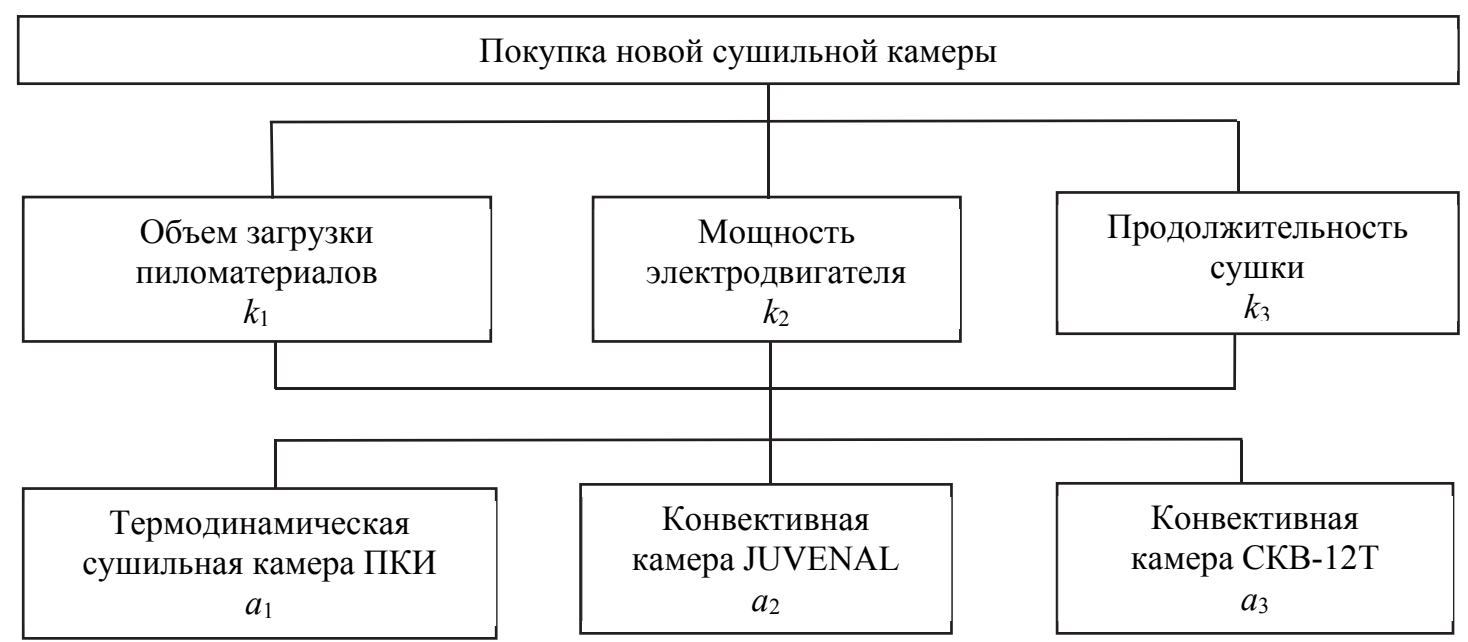

Рис. 1. Иерархия проблемы покупки сушильной камеры

Таблица 1

Индивидуальные значения критериев по выбранным альтернативам

\begin{tabular}{|l|c|c|c|}
\hline \multicolumn{1}{|c|}{ Критерий } & $\begin{array}{c}\text { Термодинамическая } \\
\text { сушильная камера ПКИ }\end{array}$ & $\begin{array}{c}\text { Конвективная камера } \\
\text { JUVENAL }\end{array}$ & $\begin{array}{c}\text { Конвективная камера } \\
\text { СКВ-12Т }\end{array}$ \\
\hline $\begin{array}{l}\text { Объем загрузки пиломате- } \\
\text { риалов } k_{1}, \mathrm{M}^{3}\end{array}$ & 36,5 & 40 & 50 \\
\hline $\begin{array}{l}\text { Мощность электродвигате- } \\
\text { ля } k_{2}, \text { кВт/ч }\end{array}$ & 20 & 14 & 5 \\
\hline $\begin{array}{l}\text { Продолжительность сушки } \\
k_{3} \text { сут. }\end{array}$ & 1,5 & 3 & 5 \\
\hline
\end{tabular}

Таблица 2

Числовые оценки матрицы попарных сравнений для критериев

\begin{tabular}{|l|c|c|c|c|c|c|}
\hline \multicolumn{1}{|c|}{ Критерии } & $k_{1}$ & $k_{2}$ & $k_{3}$ & $V$ & $W$ & $S$ \\
\hline$k_{1}$ & 1 & 3 & 0,33 & 1,0 & 0,23 & 1,0 \\
\hline$k_{2}$ & 0,33 & 1 & 0,11 & 0,33 & 0,08 & 1,04 \\
\hline$k_{3}$ & 3 & 9 & 1 & 3,0 & 0,69 & 0,99 \\
\hline Сумма & 4,33 & 13 & 1,44 & 4,33 & 1,0 & 3,03 \\
\hline \multicolumn{2}{l}{} \\
\hline
\end{tabular}


Числовые оценки матрицы парных сравнений альтернатив

(влияние $k_{1}$ - объем загрузки пиломатериалов)

\begin{tabular}{|l|c|c|c|c|c|c|}
\hline \multicolumn{1}{|c|}{ Критерии } & $a_{1}$ & $a_{2}$ & $a_{3}$ & $V$ & $W$ & $S$ \\
\hline$a_{1}$ & 1 & 0,33 & 0,11 & 0,33 & 0,06 & 0,78 \\
\hline$a_{2}$ & 3 & 1 & 0,14 & 0,75 & 0,15 & 1,25 \\
\hline$a_{3}$ & 9 & 7 & 1 & 3,98 & 0,79 & 0,99 \\
\hline Сумма & 13 & 8,3 & 1,25 & 5,06 & 1,0 & 3,02 \\
\hline \multicolumn{2}{l}{ Отношение согласованности (ОС), \% } \\
\hline \multicolumn{2}{l}{ Индекс согласованности } \\
\hline
\end{tabular}

Таблица 4

Числовые оценки матрицы парных сравнений альтернатив (влияние $k_{2}$ - мощность электродвигателя)

\begin{tabular}{|l|c|c|c|c|c|c|}
\hline \multicolumn{1}{|c|}{ Критерии } & $a_{1}$ & $a_{2}$ & $a_{3}$ & $V$ & $W$ & $S$ \\
\hline$a_{1}$ & 1 & $1 / 9$ & $1 / 3$ & 0,33 & 0,08 & 1,04 \\
\hline$a_{2}$ & 9 & 1 & 3 & 3,0 & 0,69 & 0,99 \\
\hline$a_{3}$ & 3 & $1 / 3$ & 1 & 1,0 & 0,23 & 0,99 \\
\hline Сумма & 13,0 & 1,44 & 4,33 & 4,33 & 1,0 & 3,02 \\
\hline \multicolumn{2}{l}{ Отношение согласованности (ОС), \% } \\
\hline \multicolumn{2}{l}{ Индекс согласованности } \\
\hline
\end{tabular}

Составим таблицу числовых оценок матрицы парных сравнений альтернатив по влиянию критерия $k_{3}$ (табл. 5). В табл. 5 видно, что отношение согласованности составляет $8,6 \%$.

В сводной табл. 6 представлены глобальные приоритеты, на основе которых можно сделать вывод о наиболее подходящей из предложенных альтернатив.
Исходя из величины глобальных приоритетов, можно сделать вывод, что наилучшим вариантом будет альтернатива $a_{1}$ - термодинамическая сушильная камера ПКИ, поскольку по критерию $a_{1}$ глобальный приоритет имеет наибольшее значение. Данная сушильная камера имеет оптимальное значение по времени сушки пиломатериалов, что влияет на технологию производства и позволяет производить более конкурентную продукцию.

Таблица 5

Числовые оценки матрицы парных сравнений альтернатив

(влияние $\boldsymbol{k}_{3}$ - продолжительность сушки)

\begin{tabular}{|l|c|c|c|c|c|c|}
\hline \multicolumn{1}{|c|}{ Критерии } & $a_{1}$ & $a_{2}$ & $a_{3}$ & $V$ & $W$ & $S$ \\
\hline$a_{1}$ & 1 & 3 & 9 & 3,0 & 0,67 & 0,96 \\
\hline$a_{2}$ & 0,33 & 1 & 5 & 1,18 & 0,26 & 1,09 \\
\hline$a_{3}$ & 0,11 & 0,2 & 1 & 0,28 & 0,07 & 1,05 \\
\hline Сумма & 1,44 & 4,2 & 15 & 4,46 & 1,0 & 3,1 \\
\hline \multicolumn{2}{l}{ Отношение согласованности (ОС), \% } \\
\hline
\end{tabular}

Таблица 6

Сводная таблица результатов и определения глобальных приоритетов

\begin{tabular}{|l|c|c|c|c|}
\hline \multirow{3}{*}{ Альтернативы } & \multicolumn{3}{|c|}{ Критерии } & \multirow{2}{*}{ Глобальные } \\
\cline { 2 - 3 } & $k_{1}$ & $k_{2}$ & $k_{3}$ & \\
\cline { 2 - 3 } & \multicolumn{2}{|c|}{ Писленное значение вектора приоритета } & \\
\cline { 2 - 3 } & 0,23 & 0,08 & 0,69 & 0,48 \\
\hline$a_{1}$ & 0,06 & 0,08 & 0,67 & 0,27 \\
\hline$a_{2}$ & 0,15 & 0,69 & 0,26 & 0,25 \\
\hline
\end{tabular}

Труды БГТУ Серия 6 № 12021 
Заключение. Таким образом, чтобы решить задачи выбора оборудования с учетом инновационных рисков может быть использован метод анализа иерархий.

\section{Список литературы}

1. Саати Т. Принятие решений. Метод анализа иерархий. М.: Радио и связь, 1993. 278 с.

2. Цибизова Т. Ю., Карпунин А. А. Применение МАИ в оценке качества процессов управления // Современные проблемы науки и образования. 2015. № 2. С. 200-201.

3. Водопьянова Т. П., Равино А. В., Захаренко О. А. Метод анализа иерархий как инструмент управления рисками внешнеэкономической деятельности предприятия // Труды БГТУ. Сер. 5. Экономика и управление. 2019. № 2 (226). С. 36-42.

\section{References}

1. Saati T. Prinyatiye resheniy. Metod analiza iyerarkhiy [Makingdecisions. Hierarchy Analysis Method]. Moscow, Radio i svyaz' Publ., 1993. 278 p.

2. Tsibizova T. Yu., Karpunin A. A. Application of MAI in assessing the quality of management processes. Sovremennyye problemy nauki i obrazovaniya [Modern problems of science and education], 2015, no. 2, pp. 200-201 (In Russian).

3. Vodop'yanova T. P., Ravino A. V., Zaharenko O. A. The method of analyzing hierarchies as a tool for managing the risks of foreign economic activity of an enterprise. Trudy BGTU [Proceedings of BSTU], issue 5, Ekonomics and Management, 2019, no. 2 (226), pp. 36-42 (In Russian).

\section{Информация об авторах}

Водопьянова Татьяна Павловна - кандидат экономических наук, доцент, доцент кафедры менеджмента, технологий бизнеса и устойчивого развития. Белорусский государственный технологический университет (220006, г. Минск, ул. Свердлова, 13a, Республика Беларусь). E-mail: vodopjanova@belstu.by

Жуковень Валерия Сергеевна - студентка. Белорусский государственный технологический университет (220006, г. Минск, ул. Свердлова, 13a, Республика Беларусь). E-mail: alicefrost500@gmail.com

\section{Information about the authors}

Vodop'yanova Tat'yana Pavlovna - PhD (Economics), Associate Professor, Assistant Professor, the Department of Management, Business Technology and Sustainable Development. Belarusian State Technological University (13a, Sverdlova str., 220006, Minsk, Republic of Belarus). E-mail: vodopjanova@belstu.by

Zhukoven' Valeriya Sergeyevna - student. Belarusian State Technological University (13a, Sverdlova str., 220006, Minsk, Republic of Belarus). E-mail: alicefrost500@gmail.com 